\title{
Políticas Públicas Brasileiras como Tecnologias de Saúde
}

\author{
Brazilian Public Policies as Health Technologies
}

\section{Amanda Lameck Pinho ${ }^{1}$}

Wagner Rodrigo Weinert ${ }^{2}$

\begin{abstract}
Resumo
O estudo da formação de políticas públicas nos permite entender os contextos nos quais se deram ações governamentais passadas e planejar ações futuras. Este trabalho analisa as políticas públicas na saúde como forma de tecnologia, além de explorar o âmbito da saúde no campo social. O objetivo é compreender como se constrói o debate acerca da formação de políticas públicas na saúde e sua relação com as lutas de classe entre os sujeitos sociais envolvidos, tanto na sua formulação quanto na implementação. A metodologia consistiu na pesquisa bibliográfica acerca das políticas públicas no Brasil e sua relação com a tecnologia. A conclusão encontrada reflete a influência das relações capitalistas nos debates que envolvem as estratégias de produção de políticas públicas, que acabam por reforçar os padrões sociais já pré-estabelecidos.
\end{abstract}

Palavras-chave: Políticas Públicas. Saúde. Tecnologia.

\begin{abstract}
The study of public policy's formation allows us to understand the contexts in which past governmental actions were taken and to plan future actions. This paper analyzes public policies on health as a technology, in addition to exploring the scope of health in the social field. The objective is to understand the discussion about the formation of public policies in health and the relation with the class struggles between the social subjects involved, both in its formulation and in the implementation. The methodology consisted of a bibliographical research about public policies in Brazil and its relation with technology. The conclusion reached reflects the influence of capitalist relations in the discussion that involves the strategies of public policy making, which reinforce already pre-established social patterns.
\end{abstract}

Keywords: Public Policies. Health. Technology.

\section{Introdução}

Tecnologias não se resumem apenas a artefatos, mas também podem caracterizar sistemas organizacionais. É o caso das políticas públicas. Formuladas para atender as demandas populares, são sistemas sociotécnicos decorrentes da discussão e disputa de interesses entre diversos atores

\footnotetext{
${ }^{1}$ Biomédica. Pós-graduada pelo Programa de Pós Graduação em Ciência, Tecnologia e Sociedade do Instituto Federal do Paraná - Campus Paranaguá. E-mail: pinho.1.amanda@gmail.com

${ }^{2}$ Bacharel em Informática. Professor do Programa de Pós Graduação em Ciência, Tecnologia e Sociedade do Instituto Federal do Paraná - Campus Paranaguá. E-mail: wagner.weinert@ifpr.edu.br
} 
sociais. A Policy Analysis é um instrumento de análise que desmembra tal sistema, buscando indicar os conflitos e contextos sociopolíticos relacionados nesta rede (FREY, 2000). No entanto, nem todos os atores envolvidos têm seus interesses garantidos de maneira igualitária, e isso se deve principalmente pelas lutas de classe que delimitam o lugar social de cada um. É preciso compreender as razões que levam à reivindicação popular acerca da formulação de políticas públicas e entender os fatores que fazem com que determinadas políticas sejam privilegiadas em detrimento a outras.

A origem dos estudos em políticas públicas se deu pela necessidade de entender as razões que levam o governo a tomar decisões. Esses estudos em regimes democráticos permitiram formular cientificamente e analisar tais políticas de modo a compreender seus caminhos, desde a pesquisa até sua implantação. Historicamente, a introdução de políticas públicas como ferramenta governamental surgiu na Guerra Fria, momento em que o planejamento estratégico multidisciplinar se fez necessário para a condução da guerra (SOUZA, 2006).

A formação de políticas públicas é guiada por um embate de interesses e preferências (SOUZA, 2006) entre diversos atores inseridos em uma complexa rede social. Além disso, não se pode generalizar o espaço de atuação do Estado em todas as nações, tendo em vista que este depende do contexto histórico, econômico e social enfrentado por cada país. Sendo assim, o presente trabalho enfatiza a atuação do Estado brasileiro na construção das políticas públicas. Para tanto, é necessário considerar as peculiaridades socioeconômicas e políticas brasileiras, a fim de adaptar os instrumentos de análises de políticas públicas às condições do Brasil (FREY, 2000). O conhecimento da sua própria realidade emancipa o cidadão e o capacita como detentor da construção de sua democracia, embasando e fortalecendo a participação política, necessária para a reivindicação de tomadas de ação governamentais com maior eficiência (FRIEDRICH; ALVES, 2017).

$\mathrm{Na}$ área da saúde, campo que faz do ser humano seu objeto de trabalho, torna-se imprescindível trazer para o debate os aspectos sociais que direcionam as práticas médicas, tecnologias e formulação de políticas públicas. Considerando tais questões, o presente artigo busca compreender de que forma se dão as relações no campo social das políticas públicas em saúde, sendo estas apresentadas na forma de sistemas organizacionais tecnológicos. Buscamos também compreender quais ações, entidades e atores exercem influência sobre o governo para tomada de decisões no campo da saúde e quais são as suas consequências sociais. 


\section{Desenvolvimento}

Os itens subsequentes buscam explicar o processo de construção das políticas públicas com ênfase no contexto brasileiro, bem como seus instrumentos de análise e seus principais elementos. Em seguida, analisamos sua formação no campo de saúde e a relação entre as dimensões de saúde e de sociedade e, finalmente, de que maneira as políticas públicas de saúde podem ser consideradas tecnologias dentro da metodologia de análise de rede.

\section{A construção das Políticas Públicas}

Segundo Weber (2011, p. 56), “o Estado é o único detentor da violência e tal atribuição lhe garante o monopólio da função organizacional da sociedade". Sua autonomia para estruturar as relações sociais é legitimada pela aceitação dos indivíduos que concordam em seguir as regras pré-estabelecidas pelo Estado. Com a criação do sistema capitalista, ampliaram-se as desigualdades sociais e a partir delas, fez-se necessária a criação de um sistema que beneficie a população mais empobrecida. Caberia ao Estado a responsabilidade da produção de políticas sociais de distribuição de renda para os serviços essenciais dessa população prejudicada pelo sistema (OFFE, 1975).

Segundo Offe (1975, p. 15), "a política social é a forma pela qual o Estado tenta resolver o problema da transformação duradoura do trabalho não assalariado em trabalho assalariado". Seria então uma consequência da industrialização capitalista, que mobilizou a força de trabalho e que necessitou da intervenção do Estado para realocar os trabalhadores que agora necessitavam vender sua força de trabalho. Integrando a força de trabalho no mercado, o Estado também garantia o desenvolvimento e manutenção do sistema capitalista. Aqueles que não tivessem condições de se tornarem trabalhadores deveriam então ser amparados por sistemas de proteção social. É importante, contudo, ressaltar que a política estatal não está à serviço das necessidades de qualquer grupo ou classe social, mas reage a problemas estruturais do aparelho estatal de dominação e de prestação de serviços, ou seja, do sistema capitalista (OFFE, 1975).

A construção das políticas sociais pode ser justificada pelas demandas sociais e políticas referentes a temas que reivindicam a participação da esfera estatal. Dessa forma, políticas públicas não existem em si mesmas, mas resultam de um embate de forças com interesses diversos (GONÇALVES, 2008). Segundo Merhy (2014, p. 54), os trabalhadores representam os "corpos sociais" e sua força de trabalho é o elemento vital para o capital se perpetuar. Dessa forma, quando são feitas concessões às reivindicações dos trabalhadores, deve-se considerar que seu caráter é, 
primeiramente, legitimar o Estado e, como consequência, proteger o sistema capitalista com trabalhadores dispostos a vender sua força de trabalho (OFFE, 1975).

A luta de classes também norteia as relações entre política, economia, Estado e sociedade, pois dela resultam projetos de transformação social e de hierarquização de interesses econômicos e políticos. Entender a constituição dos sujeitos sociais como forças políticas detentoras de interesses que podem ou não ser conflitantes auxilia a compreensão da formação das políticas sociais (DRAIBE, 1985).

Para estudar as políticas públicas brasileiras, é necessário compreender sua organização estrutural governamental. Esta se fundamenta na divisão municipal, estadual e estatal. Isso confere considerável independência administrativa em questões financeiras e políticas, concedendo caráter complexo, diverso e dinâmico aos estudos relacionados ao tema. Dessa forma, é necessário que as pesquisas de políticas públicas acompanhem a fragmentação institucional em cada esfera de governo (FREY, 2000). O governo federal tem importante papel na tomada de decisões, todavia os governos estaduais e municipais interferem diretamente na entrega dos serviços e sua implementação (ARRETCHE, 2012). Outra particularidade brasileira é a alta dinâmica estrutural e processual que ocorre com as transições entre governos ou até mesmo dentro de uma única gestão, fenômeno que pode influenciar diretamente nos temas de maior ou menor relevância para discussão e fomento de políticas públicas (FREY, 2000).

A Figura 1 ilustra as principais etapas presentes na construção de políticas públicas. Nele, cada fase estaria marcada pela influência dos atores integrantes da rede, bem como suas interrelações e o contexto em que estão inseridos (MARQUES, 2006). 
FIGURA 1 - PROCESSO DE PRODUÇÃO DE POLÍTICAS PÚBLICAS

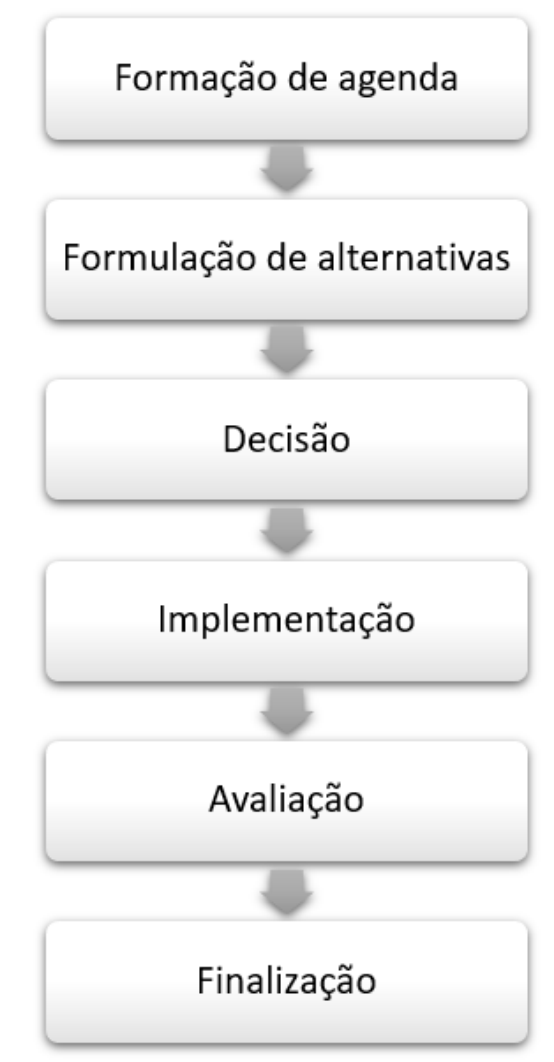

FONTE: Adaptado de PARSONS (1995).

A análise da produção de políticas públicas revela alta complexidade, principalmente no que se refere à estruturação das diversas dimensões das redes sociais, nas quais podem se inserir vínculos entre indivíduos, entidades e organizações. Compreender as redes auxilia a interpretação do contexto em que a política se desenvolve e do comportamento dos atores envolvidos (MARQUES, 2006). Segundo Frey (2000 p. 247), no Brasil "a constelação das forças sociais e políticas, ou seja, o exercício de poder pelas elites políticas e econômicas parece determinar de forma bem mais decisiva os rumos do País do que quaisquer arranjos". Percebe-se então a influência das relações de poder acerca dos acontecimentos políticos no Brasil, sendo necessária a reflexão sobre quais seriam os atores envolvidos nessas relações e seus respectivos interesses. Marques (2015) destaca a participação de quatro grupos de influência principais nas políticas urbanas: burocracias, partidos políticos, empresas privadas e movimentos sociais, e suas conexões refletem substancialmente na construção do quadro político.

O’Donnell (1991) classifica o Brasil em uma categoria de país democrata não consolidado e não institucionalizado e sugere que nessa forma de governo a influência sobre as decisões políticas 
se resume a uma elite classista. Além disso, a tomada de decisões é intermediada por partidos e associações. Segundo o autor, o agir estatal se baseia nas relações de clientelismo, priorizando interesses individuais em detrimento aos coletivos. A consequência é a não efetivação plena das políticas, mas limitadas e influenciadas pelo patrimonialismo e pela corrupção, características estas que devem ser levadas em consideração ao se investigar a formação de políticas públicas brasileiras.

Nessa teoria elitista, conforme Marques (2006), mesmo existindo uma vasta rede de atores envolvidos na disputa de resultados do processo político, devido às intensas discrepâncias de riqueza, conhecimento e status, os vencedores seriam quase sempre os mesmos, culminando em notável desproporção de poder que perpetua e estabiliza as relações hierárquicas. Tais fenômenos podem ser previstos baseado em padrões de formação de alianças que produzem os mesmos resultados políticos. Conforme Piana (2009, p. 52):

[...] as expressões da questão social, tais como: as desigualdades e as injustiças sociais são consequentes das relações de produção e reprodução social por meio de uma concentração de poder e de riqueza de algumas classes e setores dominantes, que geram a pobreza das classes subalternas. E tornam-se questão social quando reconhecidas e enfrentadas por setores da sociedade com o objetivo de transformação em demanda política e em responsabilidade pública.

Tal dificuldade em implementação de políticas sociais e conquista da cidadania refletem diretamente na saúde, setor da sociedade marcado por intensas desigualdades entre os sistemas público e privado consequente das relações capitalistas.

\section{Saúde e Sociedade}

O diagnóstico é uma das tecnologias de saúde mais utilizada. No entanto, não pode ser a única, uma vez que busca padrões de causa e efeito, nem sempre considerando as individualidades de cada paciente. Nesse contexto, uma abordagem para melhora na qualidade dos atendimentos seria a aproximação entre as dimensões de saúde e social, uma vez que entre elas existe importante fator de correlação. Benevides e Passos (2005, p. 563) apontam a humanização como estratégia de produção e otimização de saúde, considerando que: "sujeitos, quando mobilizados, são capazes de transformar realidades, transformando-se a si próprios neste mesmo processo". Essa perspectiva coloca a saúde como corresponsabilidade de vários agentes, considerando vários atores envolvidos no processo de promoção da saúde e várias ações estatais relacionadas, tais quais as reformas sanitárias. No entanto, Merhy (1992) reconhece que, isoladas, reformas sanitárias não podem ser sinônimo de políticas públicas, mas enfatiza seu caráter coletivo de intervenção e o seu papel na 
disputa por modelos tecnoassistenciais que se apresentaram como projetos de políticas para ação estatal no campo da saúde.

Buss (2000) comenta sobre a questão da desigualdade na saúde entre os países e sua influência entre regiões e grupos sociais, principalmente no que tange a condições de infraestrutura urbana básica. Estudos de Andreazzi, Barcellos e Haccon (2007) e Ferreira et al. (2006) também mostraram que existe forte associação entre as condições sanitárias e socioeconômicas em comunidades menos favorecidas, o que pode aumentar a disseminação de parasitoses nessa população mais vulnerável. Segundo Buss (2000), a principal resposta social para os problemas de saúde deve vir de investimentos em medidas preventivas, assistência médica e melhoria de condições de vida em geral.

Para redução das desigualdades sociais que refletem nas desigualdades de saúde, a criação do SUS em 1988 foi uma importante conquista. O cenário de políticas de saúde foi então reorganizado em um modelo de responsabilidade direta do setor público, em um sistema que deveria garantir serviços de saúde voltados a toda população (OBA et al., 2010). No entanto, embora possua caráter constitucional, nem todas as suas doutrinas foram plenamente implementadas. Além disso, apesar de se constituir como lei e direito, faz-se necessária a constante reinvenção do sistema na prática (BENEVIDES; PASSOS, 2005). Para Oba et al. (2010, p. 46), a construção da saúde como direito

[...] implica em compreender o cotidiano dos movimentos sociais, a constituição da demanda dos serviços de saúde, a prática institucional dos agentes envolvidos, a relação, enfim, entre os movimentos e manifestações sociais por saúde e o Estado na formulação das políticas de saúde.

A apropriação da saúde como direito envolve diversas relações, dentre elas a que acontece entre a sociedade e o Estado. Quanto mais próxima for tal relação, mais facilmente ocorre a comunicação entre os grupos organizados da sociedade e os órgãos públicos, favorecendo a articulação das demandas dos cidadãos (HOFLING, 2001). Tal formação em rede pode ser analisada sob a ótica de sistema tecnológico ao serem considerados os diversos atores envolvidos e seus respectivos interesses.

\section{Políticas Públicas em Saúde como Tecnologias}

A saúde representa a soma final de várias dimensões, dentre as quais: social, jurídica, econômica e política, dessa forma não pode ser estudada de maneira isolada desses e outros fatores. As políticas públicas em saúde exigem ações intersetoriais, por isso não é recomendável restringilas apenas na relação médico-paciente. A relevância de se discutir o papel da tecnologia nas 
relações de saúde vai além do seu uso restrito a artefatos médicos. Seu conceito abrange os medicamentos, materiais, equipamentos e procedimentos, mas também os sistemas de organização, educação, informação, dentre outros (REMONDI et al., 2017).

Nesse contexto, as políticas públicas em saúde também podem ser vistas como um sistema tecnológico segundo a definição de tecnologia social, que, segundo Costa (2013, p. 18), é o movimento que implica na "participação, empoderamento e autogestão de seus usuários [...], buscando articular a participação de gestores públicos, pesquisadores e acadêmicos, sociedade civil organizada e sociedade civil marginalizada: o movimento por tecnologias inclusivas".

A saúde pública como campo tecnológico tem como objeto de trabalho o processo coletivo de saúde-doença. Pode ser entendida como saberes tecnológicos que se concretizam em ações tecnoassistenciais (MERHY, 1992). Nesse campo, existem atores com interesses específicos e diferentes níveis de representação social. Considerando a não neutralidade da tecnologia e seus valores sociais agregados (WINNER, 1986) e as questões capitalistas que influenciam na tomada de decisões acerca das políticas públicas (BEHRING; BOSCHETTI, 2006), percebe-se que embora uma metodologia como a de "Construção Social da Tecnologia - SCOT", sugerida por Pinch e Bijker (1987), fosse apropriada para compreender como as políticas são produzidas no contexto social, ela não considera que existem atores com mais peso do que outros no debate que culmina na criação das políticas. Baptista (2015) indica a diferença entre os grupos sociais relevantes e os considerados invisíveis, sem poder de decisão. A discrepância está na influência de determinados grupos nas relações políticas e também econômicas, uma vez que a voz do capitalismo é mais ouvida que as demais, delimitando o caráter elitista das decisões político-sociais.

Examinando o caráter social da saúde, esta deve ser pensada considerando as particularidades que envolvem os diferentes segmentos populacionais, uma vez que os indivíduos se expõem de maneiras diferentes aos agravos de saúde, como os moradores de rua, populações indígenas, usuários de drogas, grupos marginalizados de centros urbanos, entre outros (BARATA, 1997). No entanto, como demonstrado no presente artigo, os fatores que norteiam a criação e implementação das políticas públicas privilegia as relações capitalistas em detrimento das sociais, transformando a medicina em um mercado no qual permanece saudável quem possui maior poder na rede de saúde na qual estamos todos inseridos.

\section{Conclusão}

As políticas públicas são a materialização do resultado da discussão entre vários atores sociais: cidadãos, políticos, instituições, organizações, dentre outros inúmeros que podem estar 
presentes ativa ou passivamente nesse campo, que vai desde a idealização até a implantação de ações que visam à conquista de um bem coletivo. A participação popular é fundamental, nesse sentido, como exercício da cidadania e estratégia de aquisição de novos benefícios sociais. Para isso, é necessário estreitar as relações entre os órgãos públicos e os grupos sociais para facilitar o processo de articulação de novas políticas públicas.

O papel do Estado é limitador nas políticas públicas, que se intensificaram no Brasil em uma fase intermediária entre o Estado mínimo e o Estado de bem-estar social. Nesse contexto, é função estatal regular, coordenar e gerenciar de maneira interdependente com o mercado. Soberano, o Estado se torna o mediador ativo de interesses, e dessa atribuição decorre o dever de prestação adequada de serviços públicos. Quando insatisfeita com a oferta do Estado, a população se organiza e reivindica novas concessões sociais, que podem ser chamadas de políticas públicas (D'ALBUQUERQUE, 2012).

O Estado deveria então representar neutralidade e imparcialidade em suas decisões. No entanto, Offe (1975) sugere que o Estado desenvolve padrões de seletividade sob a forma de arenas de decisão, formuladores de políticas públicas e temas que merecem ou não discussão nas agendas governamentais de maneira a direcionar que determinados interesses sejam garantidos, ou seja, definir os objetos das políticas públicas.

Segundo Hofling (2001 p. 39), "em um Estado de inspiração neoliberal as ações e estratégias governamentais incidem essencialmente em políticas compensatórias, em programas focalizados", indo de encontro aos apontamentos de Draibe (1993), que afirma que no Brasil os benefícios não são oferecidos à população de forma igualitária. Como consequência de tal desigualdade, as políticas são ofertadas de maneira seletiva aos mais pobres. Segundo a autora, o sistema de bemestar social brasileiro se desenvolveu na meritocracia e na estratificação, baseando-se na expansão de privilégios de classes já privilegiadas. Essa questão vai de acordo com Piana (2009), que sugere que as políticas sociais deveriam se distribuir entre todos os cidadãos, independente de sua classe social, embora saiba-se que não apenas no Brasil como na maioria dos países, tais ações manifestam-se no atendimento de políticas básicas à população excluída.

Por essa razão, existe um hiato entre a carência de determinado serviço e a implementação de políticas sociais. Barreiras como a falta de organização ou de representantes são frequentes e limitam a conquista plena dos benefícios pleiteados. Muito do que se vê são conquistas parciais, quase como esmolas, ofertadas para abafar novas manifestações populares. Nesse aspecto, as políticas públicas também podem ser vistas como produto das relações entre diferentes classes, na qual a acumulação do capital é o elemento norteador. Isso pode justificar a medicina mercantilizada voltada para a doença em vez de focar na prevenção e humanização da saúde. 
Sendo assim, os estudos acerca das políticas públicas em saúde como formas de tecnologias sociais devem considerar enxergá-las não apenas como um processo organizacional padrão, mas também observar que sua implementação é norteada principalmente pelas relações de poder que tendem a se repetir continuamente, reforçando padrões sociais já pré-estabelecidos. Para mudar o panorama e o direcionamento do processo de formação de uma política de saúde e desviar do ciclo de perpetuação do poder que se repete, é necessário analisar as redes nas quais as políticas estão inseridas, uma vez que se deve levar em conta o contexto social, econômico e político em que estão sendo implementadas para então poder identificar as entidades e atores que estão influenciando as tomadas de decisões governamentais.

\section{Referências}

ANDREAZZI, M. A. R.; BARCELLOS, C.; HACON, S. Old indicators for new problems: the relationship between sanitation and health. Revista Panamericana de Salud Pública, v. 22, n. 3, p. 211-217, 2007.

ARRETCHE, M. Democracia, federalismo e centralização no Brasil. Rio de Janeiro: FGV; Fiocruz/CEM, 2012.

BAPTISTA, V. F. Social Construction of Technology como metodologia complementar à análise de políticas públicas. Revista Contraponto, v. 1, n. 3, 2015.

BARATA, R. C. B. O desafio das doenças emergentes e a revalorização da epidemiologia descritiva. Revista de Saúde Pública, v. 31, n. 5, p. 531-537, 1997.

BEHRING, E. R.; BOSCHETTI, I. Capitalismo, liberalismo e origens da política social. In: Política Social: fundamentos e história. São Paulo: Cortez, 2006.

BENEVIDES, R.; PASSOS, E. A humanização como dimensão pública das políticas de saúde. Ciência \& Saúde Coletiva, v. 10, n. 3, 2005.

BUSS, P. M. Promoção da saúde e qualidade de vida. Ciência \& Saúde Coletiva, v. 5, n. 1, p. 163-177, 2000.

COSTA, A. B. (Org.). Tecnologia Social e Políticas Públicas. São Paulo: Instituto Pólis; Brasília: Fundação Banco do Brasil, 2013.

D'AlbuQuerque, D. M. As Agências Reguladoras e a Formulação de Políticas Públicas: uma abordagem a partir da universalização das telecomunicações por meio do Fust. Brasília: Centro Universitário de Brasília, 2012.

DRAIBE, S. Rumos e metamorfoses: um estudo sobre a constituição do Estado e as alternativas da industrialização no Brasil, 1930-1960. Rio de Janeiro: Paz e Terra, 1985. 
DRAIBE, S. Qualidade de vida e reformas de programas sociais: o Brasil no cenário Latino-americano. Lua Nova, n. 31, 1993.

FERREIRA, H.; LALA, E. R. P.; MONTEIRO, M. C.; RAIMONDO, M. L. Estudo epidemiológico localizado da frequência e fatores de risco para enteroparasitoses e sua correlação com o Estado nutricional de crianças em idade pré-escolar: Parasitoses intestinais e desenvolvimento infantil. Publicatio UEPG: Ciências Biológicas e da Saúde, v. 12, n. 4, p. 33-40, 2006.

FREY, K. Políticas Públicas: um debate conceitual e reflexões referentes à prática da análise de políticas públicas no Brasil. Planejamento e Políticas Públicas, 2000.

FRIEDRICH, D. B.; ALVES, F. D. O necessário empoderamento do cidadão à efetivação das políticas públicas no Brasil: a contribuição do capital social à efetiva participação nos instrumentos democrático-participativo-deliberativos. Revista de Direito da Cidade, v. 9, n. 2, 2017.

GONÇALVES, M. F. C. Uma Contribuição para pensar as Políticas Públicas de Educação: a burocracia como sujeito do processo de políticas públicas. Rev. Pol. Públ., São Luis, v. 12, n. 1, p. 93-98, jan./jun. 2008.

HOFLING, E. M. Estado e Políticas (Públicas) Sociais. Cadernos Cedes, ano XXI, n. 55, 2001.

MARQUES, E. C. Estado, atores políticos e governança. FERNANDES, A. C.; LACERDA, N.; PONTUAL, V. (Orgs.). Desenvolvimento, planejamento e governança: expressões do debate contemporâneo. Rio de Janeiro: Letra Capital/ANPUR, 2015. p. 181-201.

MARQUES, E. C. Redes Sociais e Poder no Estado Brasileiro - Aprendizados a partir das políticas urbanas. Revista Brasileira de Ciências Sociais, v. 21, n. 60, 2006.

MERHY, E. E. A saúde pública como política. São Paulo: Hucitec, 1992.

MERHY, E. E. Capitalismo e a saúde pública: a emergência das práticas sanitárias no estado de São Paulo. 2. ed. Porto Alegre: Rede UNIDA, 2014.

OBA, M. V.; PINTO, M. C. R. L. R.; SOUZA, M. G. A. A tecnologia e a organização social das práticas de assistência à saúde da mulher. J Health Sci Inst, v. 28, n. 1, p. 42-46, 2010.

O’DONNELL, Guillermo. Democracia Delegativa? Novos Estudos, n. 31, p. 25-40, 1991.

OFFE, C. Teoria do Estado e Política social. Problemas estruturais do Estado capitalista. Rio de Janeiro: Tempo Brasileiro, 1975.

PARSONS, W. Public policy: an introduction to the theory and practice of policy analysis. Londres: Edward Elgar Pub., 1995.

PIANA, M. C. A construção do perfil do assistente social no cenário educacional [online]. São Paulo: Cultura Acadêmica, 2009.

PINCH, T.; BIJKER, W. E. The social construction of facts and artifacts: or how the Sociology of Science and the Sociology of Technology might benefit each other. Cambridge, The Mit Press, 1987. 
REMONDI, F.; LAZAROTTO, H.; ALMEIDA, M.; CAPUTO NETO, M. A Gestão e a Avaliação de Tecnologias em Saúde no Paraná: desafios e propostas para a SESA e para o setor de saúde paranaense. Revista De Saúde Pública Do Paraná, Londrina, v. 18. n. 1, jul. 2017.

SOUZA, C. Políticas Públicas: uma revisão da literatura. Sociologias, Porto Alegre, v. 8, n. 16, p. 20-45, jul./dez. 2006.

WEBER, M. A Política como Vocação. In: Ciência e Política - Duas Vocações. $18^{\circ}$ ed. São Paulo: Cultrix, 2011.

WINNER, L. Do Artifacts have Politics? The Whale and the Reactor - A Search for Limits in an Age of High Technology. Chicago: The University of Chicago Press, 1986. p. 19-39.

Artigo recebido em 13/04/2020. Aceito para publicação em 19/06/2020. 